\title{
Aplicação de formononetina na colonização e esporulação de fungos micorrízicos em braquiária
}

\author{
Cândido Barreto de Novais ${ }^{(1)}$ e José Oswaldo Siqueira(1) \\ (1)Universidade Federal de Lavras, Departamento de Ciências do Solo, Caixa Postal 37, CEP 37200-000 Lavras, MG. E-mail: candidobnn@yahoo.com.br, \\ siqueira@cnpq.br
}

Resumo - O objetivo deste trabalho foi avaliar o efeito de aplicações de Mycoform na colonização micorrízica e esporulação de 13 isolados de fungos micorrízicos arbusculares em Brachiaria decumbens. O experimento foi conduzido em casa de vegetação, em solo esterilizado, com delineamento experimental inteiramente casualizado, em arranjo fatorial 3x13, com cinco repetições. O produto foi aplicado no plantio e foi ou não aplicado uma segunda vez 60 dias depois, na quantidade de $2 \mathrm{mg} \mathrm{kg}^{-1}$ de solo. Aos 150 dias de crescimento das plantas, foram coletadas amostras de raízes e de solo rizosférico, para a avaliação de colonização radicular e densidade de esporos. Houve estímulo do Mycoform nos parâmetros avaliados, efeito que variou com os isolados estudados. Foi observado efeito significativo da aplicação do Mycoform na colonização das raízes pelos isolados Glomus clarum DCS 09 e DCS 10, Paraglomus occultum DCS 06 e Acaulospora delicata DCS 02 e na esporulação dos isolados G. clarum DCS 09 e DCS 10, P. occultum DCS 06 e DCS 31, Glomus etunicatum DCS 12, A. delicata DCS 30 e Kuklospora colombiana DCS 03. O incremento na esporulação atingiu $89 \%$ e, na colonização, $60 \%$, o que confirma os benefícios da formononetina na colonização e na esporulação dos fungos micorrízicos arbusculares.

Termos para indexação: Brachiaria decumbens, estimulante de micorriza, multiplicação de FMAs, Mycoform, simbiose radicular.

\section{Formononetin application on colonization and sporulation of arbuscular mycorrhizal fungi in Brachiaria}

\begin{abstract}
The aim of this work was to evaluate the effects of Mycoform on both sporulation and colonization of 13 arbuscular mycorrhiza fungi (AMF) isolates in Brachiaria decumbens. The experiment was carried out in greenhouse conditions with sterile soil, in a completely randomized design with $3 \times 13$ factorial treatments and five repetitions. The product was applied once at planting and was or was not applied a second time 60 days afterwards, at an amount of $2 \mathrm{mg} \mathrm{kg}^{-1}$ of soil. Plants were allowed to grow for 150 days, when root and rhizospheric soil samples were collected to evaluate the percentage of colonized root segments and the spore density. Mycoform effects were different among the fungal isolates. It had significant effect on colonization for the isolates Glomus clarum DCS 09 and DCS 10, Paraglomus occultum DCS 06 and Acaulospora delicata DCS 02, and on spore density for the isolates G. clarum DCS 09 and DCS 10, P. occultum DCS 06 and DCS 31 , Glomus etunicatum DCS 12, A. delicata DCS 30 and Kuklospora colombiana DCS 03. Maximum increases due to Mycoform were $60 \%$ for colonization and $89 \%$ for spore density. These results confirm formononetin effects on AMF colonization and on AMF sporulation.
\end{abstract}

Index terms: Brachiaria decumbens, mycorrhizal stimulate, AMF multiplication, Mycoform, root symbiosis.

\section{Introdução}

A produção agrícola sustentável está associada ao desenvolvimento de tecnologias que minimizem os impactos ambientais e que poupem insumos não renováveis, como os fertilizantes a base de fósforo. Nesse sentido, há grande interesse em estudos sobre a microbiota do solo, que apresenta papel fundamental na manutenção da fertilidade dos solos agrícolas e contribui para a sua sustentabilidade. Entre os componentes da microbiota do solo, merecem destaque aqueles que formam associações mutualistas com as raízes, como os glomeromicetos, que desempenham funções significativas para o crescimento e desenvolvimento das plantas (Parniske, 2008). Esses fungos formam simbiose denominada micorriza arbuscular com mais de $80 \%$ das espécies vegetais, o que os torna componentes essenciais para a funcionalidade $\mathrm{e}$ 
manutenção dos ecossistemas naturais e manejados (Fitter, 2005; Moreira \& Siqueira, 2006).

Apesar do grande volume de estudos sobre os fungos micorrízicos arbusculares (FMAs) e sua simbiose, o desenvolvimento tecnológico para uso comercial desses simbiontes tem sido bastante limitado, por serem eles biotróficos obrigatórios. O atual nível de conhecimento técnico possibilita três estratégias principais para explorar esses fungos: inoculação com espécies fúngicas selecionadas; manejo das populações nativas (Souza et al., 1999); aplicação de produtos capazes de estimular e acelerar a colonização das raízes por fungos indígenas (Siqueira et al., 2002). Esta última estratégia apresenta grande potencial de aplicação na agricultura extensiva e pode ser eficaz também para a produção massal de esporos por indústrias de inoculantes. Flavonoides em geral podem estimular a germinação de esporos (Baptista \& Siqueira, 1994), a colonização (Nair et al., 1991; Siqueira et al., 1991a) e o crescimento e produção vegetal (Silva-Júnior \& Siqueira, 1997, 1998; Davies Junior et al., 2005a, 2005b), mas nada se conhece sobre o efeito dessas substâncias na esporulação.

Como os FMAs são organismos benéficos para os vegetais e, portanto, de grande interesse agronômico, devem ser produzidos em larga escala, a fim de possibilitar a sua aplicação e a consequente redução dos insumos agrícolas (Bagyaraj \& Reddy, 2005). No Brasil, não existe nenhum registro de inoculante para FMAs, o que torna o campo de pesquisa para produção e comercialização de inóculos bastante promissor. O inóculo de FMAs é produzido apenas em pequena escala, por universidades e institutos de pesquisa. A produção de inoculantes micorrízicos normalmente utiliza a multiplicação de FMAs em vasos com os mais variados tipos de plantas hospedeiras. As gramíneas são as mais utilizadas, e são cultivadas em substratos ou misturas de substratos - areia, solo, vermiculita, turfa, entre outros - (Gianinazzi \& Vosátka, 2004). Outros sistemas de produção de inoculante utilizados são o aeropônico (Mohammad et al., 2000) e o hidropônico, que permitem a produção de raízes colonizadas e de esporos. Técnicas mais recentes têm possibilitado a produção de inóculo a partir de raízes transformadas para a obtenção de cultura monospórica de FMA in vitro (Schubert \& Lubraco, 2000; Declerck et al., 2005). No entanto, mesmo com várias pesquisas no campo de produção de inóculo de FMAs, não se consegue propagar o fungo axenicamente (Douds Junior et al., 2006). Mesmo as espécies de fácil multiplicação muitas vezes apresentam esporulação reduzida, o que dificulta a obtenção de inoculante para estudos ou aplicação (Powell \& Bagyaraj, 1984). Por isso, procedimentos capazes de estimular a esporulação são de grande interesse.

Embora os mecanismos que regulam a esporulação dos FMAs não sejam conhecidos, existem evidências da ocorrência de resposta positiva ou negativa da espécie de planta hospedeira com diferentes FMAs, o que faz com que alguns produzam grande número de esporos e outros mal se multipliquem (Bever, 2002). Além disso, é necessário que ocorra uma colonização mínima para que o fungo possa completar seu ciclo de vida e esporular. Assim, espera-se que substâncias capazes de estimular a colonização micorrízica, como o isoflavonoide formononetina (Nair et al., 1991; Siqueira et al., 1991a), possam também estimular a produção de esporos e, desse modo, facilitar a aplicação dos FMAs na agricultura. Apesar de vários trabalhos já terem avaliado os efeitos da formononetina na colonização e crescimento vegetal (Silva-Júnior \& Siqueira, 1997, 1998; Davies Junior et al., 2005a, 2005b), o efeito dessas substâncias na esporulação de diferentes espécies de FMAs ainda não foi devidamente avaliado.

O objetivo deste trabalho foi avaliar o efeito de aplicações de Mycoform, um produto comercial a base do isoflavonoide formononetina (7-hidroxi, 4'-metoxi isoflavona), na colonização micorrízica e na esporulação de 13 isolados de FMA em Brachiaria decumbens.

\section{Material e Métodos}

O experimento foi realizado em casa de vegetação no Departamento de Ciência do Solo da Universidade Federal de Lavras, durante 150 dias. O substrato empregado foi uma mistura preparada com um Latossolo Vermelho distrófico - coletado em área sob fragmento de mata nativa no campus da Ufla, na camada superficial $(0-20 \mathrm{~cm})$ - e areia lavada, na proporção de 2:1 (v/v). Antes da mistura, o solo foi incubado com calcário dolomítico (PRNT 100\%), para elevar a saturação por bases para $60 \%$. Em seguida, foram aplicados $50 \mathrm{mg}$ de fosfato de rocha por quilograma de solo. $\mathrm{O}$ substrato foi tratado com brometo de metila ( $98 \%$ de brometo de metila e $2 \%$ de 
cloropicrina) na dosagem de $393 \mathrm{~cm}^{3} \mathrm{~m}^{-3}$, para eliminar os propágulos de FMAs nativos. Análises realizadas em amostras do substrato após a correção e adubação apresentaram as seguintes características químicas: $\mathrm{pH}$ (água) 5,$7 ; \mathrm{Ca}^{2+}, 3,4 \mathrm{cmol}_{\mathrm{c}} \mathrm{dm}^{-3} ; \mathrm{Mg}^{2+}, 1,0 \mathrm{cmol}_{\mathrm{c}} \mathrm{dm}^{-3}$; $\mathrm{Al}^{3+}, \quad 0,0 \quad \mathrm{cmol}_{\mathrm{c}} \quad \mathrm{dm}^{-3} ; \mathrm{H}^{+}+\mathrm{Al}^{3+}, \quad 5,6 \mathrm{cmol}_{\mathrm{c}} \mathrm{dm}^{-3}$; $\mathrm{P}, 7,8 \mathrm{mg} \mathrm{dm}^{-3}$ (Mehlich 1); K, $12 \mathrm{mg} \mathrm{dm}^{-3}$ (Mehlich 1); $\mathrm{V}, 44,2 \%$ e $\mathrm{MO}, 4,8 \mathrm{dag} \mathrm{kg}^{-1}$.

Após o preparo, o substrato foi distribuído em vasos de $0,5 \mathrm{dm}^{3}$. Cada vaso recebeu $40 \mathrm{~mL}$ de uma solução de Mycoform - formulação mais solúvel da formononetina, desenvolvida pela PHC, Inc., que contém $83 \%$ de fomononetina ativa (PHC, Inc., Pittsburgh, EUA) previamente preparada pela diluição de $200 \mathrm{mg}$ de Mycoform em $8 \mathrm{~L}$ de água ligeiramente aquecida (máximo de $40^{\circ} \mathrm{C}$ ), o que resultou no fornecimento de $2 \mathrm{mg}$ de Mycoform por quilograma de solo. A planta hospedeira utilizada foi Brachiaria decumbens Stapf. As sementes de $B$. decumbens foram escarificadas por meio de imersão em ácido sulfúrico concentrado comercial por 3 min e lavagem em água corrente para eliminar todo o ácido. Em seguida, foram germinadas em vermiculita estéril. As plântulas foram transplantadas para os vasos de cultivo no momento da implantação do experimento. Foram plantadas quatro plântulas por vaso. A inoculação foi realizada nas raízes no ato da transferência das plântulas, pela aplicação de uma suspensão de esporos, calibrada de modo a fornecer aproximadamente 200 esporos por vaso.

Os tratamentos consistiram da aplicação de $2 \mathrm{mg} \mathrm{kg}^{-1}$ de solo de Mycoform no ato do transplantio (Myc 1) e 60 dias depois (Myc 2) e um controle (Myc 0), e de 13 isolados de FMAs disponíveis na coleção da Universidade Federal de Lavras (Tabela 1).
O experimento foi disposto em delineamento experimental inteiramente casualizado, em arranjo fatorial 3x13, com cinco repetições. Após 150 dias de crescimento, as plantas foram retiradas dos vasos, e as raízes foram separadas. Após lavagem, foi retirado aproximadamente $1 \mathrm{~g}$ de raízes frescas de cada planta para coloração (Phillips \& Hayman, 1970) e posterior avaliação da percentagem de colonização (Giovannetti \& Mosse, 1980). Em seguida, o substrato foi seco à sombra e homogeneizado, embalado em saco de plástico e armazenado em câmara fria a $4^{\circ} \mathrm{C}$, para posterior extração ( $25 \mathrm{~mL}$ de solo) e contagem dos esporos em microscópio estereoscópico. Os dados foram submetidos a análise de correlação de Pearson, análise de variância e teste de média (Scott-Knott) pelo SISVAR (Ferreira, 2006).

\section{Resultados e Discussão}

Foram observados efeitos significativos $(p<0,05)$ da aplicação do Mycoform na colonização e na esporulação dos FMAs (Tabela 2), e esses efeitos variaram de acordo com os isolados testados. $\mathrm{Na}$ ausência do Mycoform, as plantas com os isolados $P$. occultum DCS 24 e K. colombiana DCS 03 foram as que atingiram as maiores percentagens de colonização e não responderam à aplicação do produto. A combinação desses isolados com a planta apresentou elevada compatibilidade, e não houve potencial para o estímulo adicional à colonização provocado pelo princípio ativo do Mycoform. De acordo com Siqueira et al. (1991a), o efeito do isoflavonoide formononetina é desprezível em condições que maximizam a colonização. Por outro lado, o isolado P. occultum DCS 06, que apresentou

Tabela 1. Isolados da coleção de fungos micorrízicos arbusculares da Universidade Federal de Lavras utilizados no estudo.

\begin{tabular}{lll}
\hline Espécie & $\begin{array}{c}\text { Código de } \\
\text { acesso }\end{array}$ & Origem/cultura \\
\hline Acaulospora delicata C. Walker, C.M. Pfeiff. \& Bloss (1986) & DCS 02 & Poços de Caldas, MG - Áreas de mineração \\
Acaulospora delicata C. Walker, C.M. Pfeiff. \& Bloss (1986) & DCS 30 & Capim-gordura \\
Acaulospora morrowiae Spain \& N.C. Schenck (1984) & DCS 23 & Áreas contaminadas com metais - Gramíneas \\
Glomus clarum T.H. Nicolson \& N.C. Schenck (1979) & DCS 09 & Universidade da Flórida - Brachiaria decumbens \\
Glomus clarum T.H. Nicolson \& N.C. Schenck (1979) & DCS 10 & Três Pontas, MG - Cafeeiro \\
Glomus etunicatum W.N. Becker \& Gerd. (1977) & DCS 12 & São S. do Paraíso, MG - Cafeeiro \\
Glomus etunicatum W.N. Becker \& Gerd. (1977) & DCS 13 & Três Pontas, MG - Cafeeiro \\
Paraglomus occultum (C. Walker) J.B. Morton \& D. Redecker (2001) & DCS 06 & Amazonas - Pastagem \\
Paraglomus occultum (C. Walker) J.B. Morton \& D. Redecker (2001) & DCS 24 & Amazonas - Pastagem \\
Paraglomus occultum (C. Walker) J.B. Morton \& D. Redecker (2001) & DCS 32 & Gramíneas \\
Paraglomus occultum (C. Walker) J.B. Morton \& D. Redecker (2001) & DCS 31 & Amazonas - Floresta \\
Kuklospora colombiana (Spain \& N.C. Schenck) Oehl \& Sieverd. (2006) & DCS 03 & Cerrado natural \\
Fuscutata heterogama Oehl et al. (2008) & DCS 19 & Universidade da Flórida - Brachiaria decumbens \\
\hline
\end{tabular}

Pesq. agropec. bras., Brasília, v.44, n.5, p.496-502, maio 2009 
baixa colonização na ausência do produto, respondeu positivamente à sua aplicação com um incremento de $60 \%$ na colonização. O Mycoform também teve efeito significativo na colonização das plantas pelos fungos A. delicata DCS 02, G. clarum DCS 09 e G. clarum DCS 10, porém com incremento menor, de 25 a $36 \%$, em relação ao controle. O efeito significativo do Mycoform na colonização foi, portanto, restrito a apenas quatro dos isolados fúngicos estudados. SilvaJúnior \& Siqueira (1998) verificaram que a aplicação de formononetina aumenta a micorrização da soja com Acaulospora morrowiae, G. clarum DCS 10, G. etunicatum e Fuscutata heterogama. Destes, apenas o isolado G. clarum DCS 10 respondeu positivamente à aplicação desse isoflavonoide, com um incremento médio de 36\% na colonização de $B$. decumbens.

O número de esporos extraídos do solo também diferiu entre os isolados e tratamentos. Os isolados G. clarum DCS 09 e DCS 10 apresentaram as maiores esporulações, enquanto os isolados $F$. heterogama DCS 19, G. etunicatum DCS 12 e A. morrowiae DCS 23 foram os que menos esporularam, independentemente da presença de Mycoform. Foi observado efeito positivo do Mycoform em sete isolados: K. colombiana DCS 03, P. occultum DCS 06, G. clarum DCS 09, G. clarum DCS 10, G. etunicatum DCS 12, A. delicata DCS 30 e P. occultum DCS 31 (Tabela 3). O isolado P. occultum

Tabela 2. Efeito da aplicação de Mycoform sobre a colonização micorrízica (\%) de Brachiaria decumbens submetida à inoculação de diferentes isolados de fungos micorrízicos arbusculares (FMAs) aos 150 dias após o plantio.

\begin{tabular}{lccc}
\hline Isolados de FMAs & \multicolumn{3}{c}{ Percentagem de colonização ${ }^{(1)}$} \\
\cline { 2 - 4 } Acaulospora delicata - DCS 02 & Myc0 & Myc1 & Myc2 \\
Acaulospora delicata - DCS 30 & $52 \mathrm{Ba}$ & $60 \mathrm{Ba}$ & $60 \mathrm{Ba}$ \\
Acaulospora morrowiae - DCS 23 & $51 \mathrm{Ba}$ & $54 \mathrm{Ba}$ & $64 \mathrm{Ba}$ \\
Glomus clarum - DCS 09 & $54 \mathrm{Bb}$ & $71 \mathrm{Aa}$ & $71 \mathrm{Aa}$ \\
Glomus clarum - DCS 10 & $55 \mathrm{Bb}$ & $69 \mathrm{Aa}$ & $75 \mathrm{Aa}$ \\
Glomus etunicatum - DCS 12 & $54 \mathrm{Ba}$ & $63 \mathrm{Aa}$ & $63 \mathrm{Ba}$ \\
Glomus etunicatum - DCS 13 & $55 \mathrm{Ba}$ & $59 \mathrm{Ba}$ & $60 \mathrm{Ba}$ \\
Paraglomus occultum - DCS 06 & $38 \mathrm{Db}$ & $61 \mathrm{Ba}$ & $55 \mathrm{Ca}$ \\
Paraglomus occultum -DCS 24 & $61 \mathrm{Aa}$ & $57 \mathrm{Ba}$ & $58 \mathrm{Ba}$ \\
Paraglomus occultum - DCS 32 & $52 \mathrm{Ba}$ & $54 \mathrm{Ba}$ & $53 \mathrm{Ca}$ \\
Paraglomus occultum - DCS 31 & $53 \mathrm{Ba}$ & $53 \mathrm{Ba}$ & $59 \mathrm{Ba}$ \\
Kuklospora colombiana - DCS 03 & $60 \mathrm{Aa}$ & $66 \mathrm{Aa}$ & $70 \mathrm{Aa}$ \\
Fuscutata heterogama - DCS 19 & $45 \mathrm{Ca}$ & $47 \mathrm{Ba}$ & $41 \mathrm{Da}$ \\
\hline Média & 52 & 59 & 61 \\
\hline (1) Myc0, ausência de Mycoform; Myc1, uma aplicação de Mycoform; \\
Myc2, duas aplicações de Mycoform; médias seguidas de letras iguais, \\
maiúsculas na coluna e minúsculas na linha, não diferem entre si pelo teste \\
de Skott-Knott, a de 5\% de probabilidade.
\end{tabular}

DCS 31 respondeu somente com a segunda aplicação, com densidade de esporos $38 \%$ superior ao controle. Glomus clarum DCS 10, G. etunicatum DCS 12 e P. occultum DCS 06 responderam à primeira aplicação de Mycoform e não se beneficiaram da aplicação adicional do produto. O isolado K. colombiana DCS 03 , embora não tenha apresentado resposta à aplicação do produto em termos de colonização, mostrou elevada resposta na densidade de esporos (incremento de $89 \%$ ), o que sugere a existência de mecanismo distinto da formononetina na colonização e esporulação.

Os graus de colonização e esporulação variaram entre os isolados geográficos de mesma espécie, conforme pode ser observado para $P$. occultum, em que apenas os isolados DCS 06 e DCS 31 responderam ao Mycoform para colonização e esporulação. Fato semelhante foi observado entre os isolados de G. etunicatum, nos quais a primeira aplicação foi suficiente para promover um aumento de $86 \%$ no número de esporos do isolado DCS 12, enquanto no DCS 13 nem mesmo duas aplicações foram suficientes para estimular a esporulação em $B$. decumbens. Deve-se ressaltar que G. etunicatum DCS 13 apresentou elevada esporulação mesmo na ausência do Mycoform, e essa é uma possível explicação para a ausência de resposta à aplicação do produto nessa espécie. O efeito estimulante da formononetina parece se limitar a condições de baixo potencial de inóculo inicial (Siqueira et al., 1991a). De maneira geral, o comportamento diferenciado de isolados específicos de FMAs tem sido encontrado onde

Tabela 3. Produção média de esporos de diferentes isolados de fungos micorrízicos arbusculares (FMAs) em Brachiaria decumbens na ausência (Myc0) e presença de uma (Myc1) ou duas (Myc2) aplicações de Mycoform.

\begin{tabular}{lrrr}
\hline Isolados de FMAs & \multicolumn{3}{c}{ Número de esporos ${ }^{(1)}$} \\
\cline { 2 - 4 } & $\mathrm{Myc} 0$ & \multicolumn{1}{c}{$\mathrm{Myc} 1$} & $\mathrm{Myc} 2$ \\
\hline Acaulospora delicata - DCS 02 & $1.289 \mathrm{Ba}$ & $1.178 \mathrm{Da}$ & $1.775 \mathrm{Ba}$ \\
Acaulospora delicata - DCS 30 & $1.558 \mathrm{Bb}$ & $2.071 \mathrm{Ba}$ & $2.740 \mathrm{Aa}$ \\
Acaulospora morrowiae - DCS 23 & $465 \mathrm{Ca}$ & $776 \mathrm{Da}$ & $782 \mathrm{Da}$ \\
Glomus clarum - DCS 09 & $2.264 \mathrm{Ab}$ & $3.358 \mathrm{Aa}$ & $3.108 \mathrm{Aa}$ \\
Glomus clarum - DCS 10 & $2.552 \mathrm{Ab}$ & $3.317 \mathrm{Aa}$ & $2.288 \mathrm{Ab}$ \\
Glomus etunicatum - DCS 12 & $938 \mathrm{Cb}$ & $1.753 \mathrm{Ca}$ & $954 \mathrm{Cb}$ \\
Glomus etunicatum - DCS 13 & $1.310 \mathrm{Ba}$ & $1.453 \mathrm{Ca}$ & $1.204 \mathrm{Ca}$ \\
Paraglomus occultum - DCS 24 & $1.288 \mathrm{Ba}$ & $991 \mathrm{Da}$ & $1.326 \mathrm{Ca}$ \\
Paraglomus occultum - DCS 32 & $1.703 \mathrm{Ba}$ & $1.566 \mathrm{Ca}$ & $1.460 \mathrm{Ca}$ \\
Paraglomus occultum - DCS 31 & $1.579 \mathrm{Bb}$ & $1.005 \mathrm{Db}$ & $2.189 \mathrm{Ba}$ \\
Kuklospora colombiana - DCS 03 & $1.315 \mathrm{Bb}$ & $2.490 \mathrm{Ba}$ & $2.072 \mathrm{Ba}$ \\
Fuscutata heterogama - DCS 19 & $260 \mathrm{Ca}$ & $312 \mathrm{Da}$ & $274 \mathrm{Da}$ \\
\hline Média do número de esporos & 1.393 & 1.776 & 1.657 \\
\hline (1)Médias seguidas de letras iguais, maiúsculas na coluna e minúsculas na linha, \\
não diferem entre si pelo teste de Skott-Knott, a de 5\% de probabilidade.
\end{tabular}

Pesq. agropec. bras., Brasília, v.44, n.5, p.496-502, maio 2009 
a formononetina estimula algumas espécies de FMAs (Nair et al., 1991; Siqueira et al., 1991a, 1991b; SilvaJúnior \& Siqueira, 1998), porém é necessário entender a relação do número de propágulos infectivos inicial com a resposta à aplicação de formononetina. Além disso, em alguns casos, a aplicação da formononetina pode apresentar-se como inibidora de G. etunicatum, G. macrocarpum (Tsai \& Phillips, 1991) e G. rosea (Bécard et al., 1992; Chabot et al., 1992).

Não foi encontrada, no presente trabalho, relação entre nível de colonização e esporulação nos isolados na ausência de Mycoform. Porém, quando o produto foi aplicado, mesmo com o efeito diferenciado dos isolados houve boa relação entre colonização e a densidade de esporos no solo (Figura 1). Portanto, a aplicação de formononetina altera o padrão de esporulação e a interdependência desta com o grau de colonização dos respectivos isolados. Dos 13 isolados testados, destacaram-se como os de maior potencial responsivo ao Mycoform os isolados A. delicata DCS 30, G. etunicatum DCS 12, K. colombiana DCS 03, P. occultum DCS 06 e P. occultum DCS 31, cujos aumentos na esporulação foram elevados, tendo atingido quase $90 \%$. Ao se considerar o efeito médio significativo da aplicação de Mycoform nos isolados com resposta positiva, observa-se que a produção de esporos por estes isolados passou de uma média de 1.685 para 2.666 esporos por $50 \mathrm{~mL}$ de solo, o que corresponde a um aumento de 39 esporos por $\mathrm{mL}$ de solo avaliado.

O presente trabalho confirma o efeito estimulante do isoflavonoide formononetina na colonização, como já foi observado em vários estudos (Nair et al., 1991; Siqueira et al., 1991a, 1991b), e, pela primeira vez, constata o efeito benéfico da aplicação desse isoflavonoide na esporulação de FMAs. Davies Junior et al. (2005b) também encontraram aumento no número de esporos de FMAs indígenas pertencentes aos gêneros Gigaspora e Glomus, quando aplicado o isoflavonoide formononetina formulado como Myconate, o que resultou em aumento na atividade micorrízica e na produtividade de batata. Não encontraram efeito para Scutellospora sp., que também não respondeu ao Mycoform no presente trabalho.

Embora o efeito do Mycoform sobre os FMAs não seja generalizado, esse produto tem potencial para promover uniformização e aumento da produção de esporos. Como a densidade de esporos é geralmente baixa em solos agrícolas, a aplicação de produtos à base de formononetina pode contribuir para aumentar os benefícios dos FMAs para a produção agrícola.
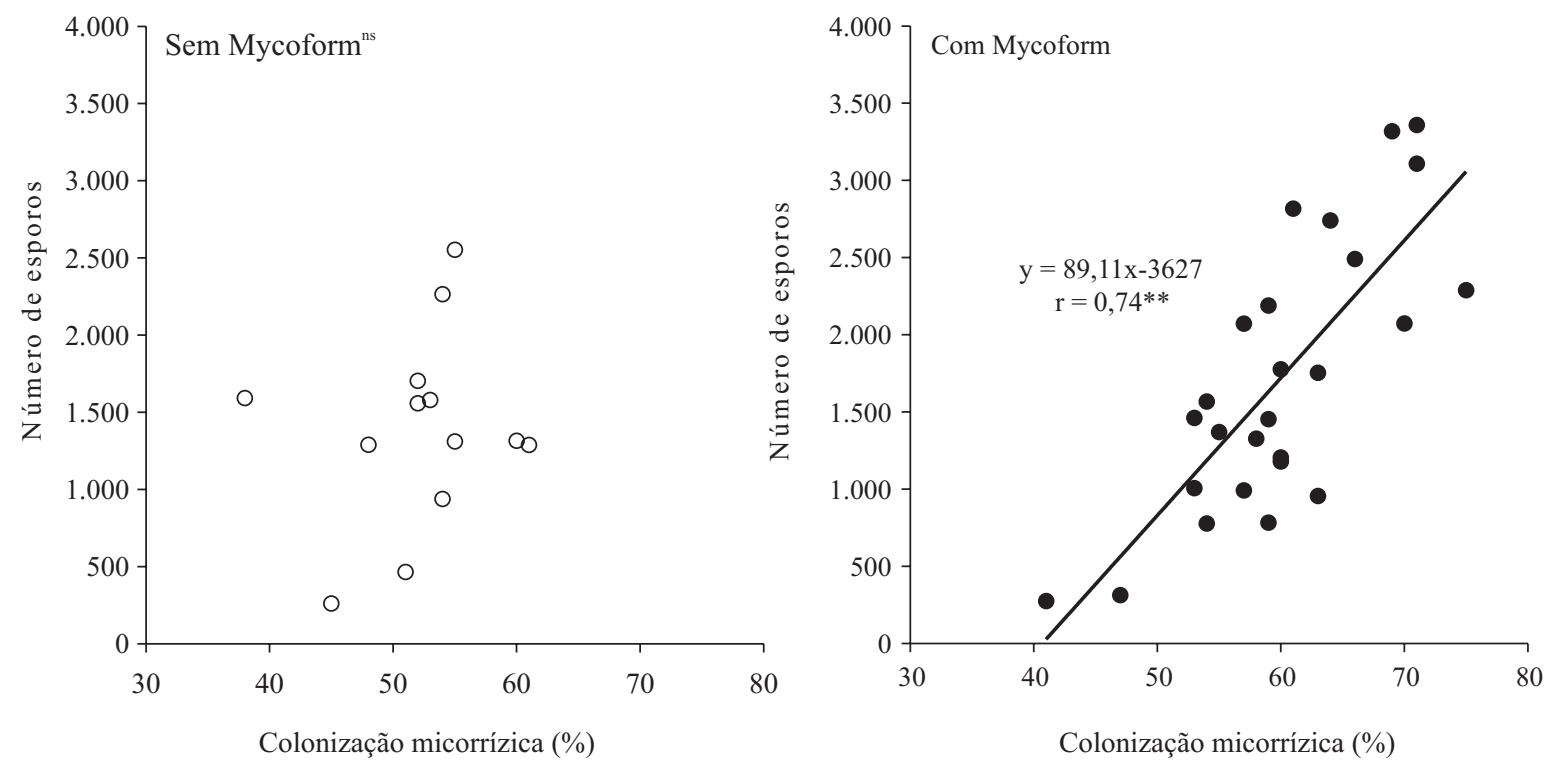

Figura 1. Relação entre a colonização micorrízica e a produção de esporos por diferentes isolados com e sem a aplicação de Mycoform. ${ }^{\text {ns }}$ Não significativo. $* *$ Significativo a $1 \%$ de probabilidade pela análise de correlação de Pearson. 


\section{Conclusões}

1. A aplicação do Mycoform aumenta a colonização e a esporulação dos fungos micorrízicos arbusculares (FMAs) em Brachiaria decumbens, e o efeito varia de acordo com o isolado fúngico.

2. A aplicação do Mycoform altera a relação entre a colonização e a esporulação dos FMAs, e torna essas variáveis mais inter-relacionadas.

3. O efeito do Mycoform na colonização e esporulação é maior nos isolados de atividade mais baixa.

\section{Agradecimentos}

Ao Conselho Nacional de Desenvolvimento Científico e Tecnológico, pelo financiamento da pesquisa; à Coordenação de Aperfeiçoamento de Pessoal de Nível Superior, pela concessão de bolsa.

\section{Referências}

BAGYARAJ, D.J.; REDDY, B.J.D. Application of arbuscular mycorrhizal fungi in horticulture. In: MEHROTRA, V.S. (Ed). Mycorrhiza: role and applications. New Dehli: Allied Publisher, 2005. p.237-254.

BAPTISTA, M.J.; SIQUEIRA, J.O. Efeito de flavonóides na germinação de esporos e no crescimento assimbiótico do fungo micorrízico arbuscular Gigaspora gigantea. Revista Brasileira de Fisiologia Vegetal, v.6, p.127-134, 1994.

BÉCARD, G.; DOUDS, D.D.; PFEFFER, P.E. Extensive in vitro hyphal growth of vesicular-arbuscular mycorrhizal fungi in the presence of $\mathrm{CO}_{2}$ and flavonols. Applied and Environmental Microbiology, v.58, p.821-825, 1992.

BEVER, J.D. Host-specificity of AM fungal population growth rates can generate feedback on plant growth. Plant and Soil, v.244, p.281-290, 2002.

CHABOT, S.; BEL-RHID, R.; CHÊNEVERT, R.; PICHÉ, Y. Hyphal growth promotion in vitro of the VA mycorrhizal fungus, Gigaspora margarita Becker \& Hall, by the activity of structurally specific flavonoid compounds under $\mathrm{CO}_{2}$ enriched conditions. New Phytologist, v.122, p.461-467, 1992.

DAVIES JUNIOR, F.T.; CALDERÓN, C.M.; HUAMAN, Z. Influence of arbuscular mycorrhizae indigenous to Peru and a flavonoid on growth, yield and leaf elemental concentration of 'Yungay' potatoes (Solanum tuberosum L.). HortScience, v.40, p.381-385, 2005a.

DAVIES JUNIOR, F.T.; CALDERÓN, C.M.; HUAMAN, Z.; GÓMEZ, R. Influence of a flavonoid (formononetin) on mycorrhizal activity and potato crop productivity in the highlands of Peru. Scientia Horticulturae, v.106, p.318-329, 2005 b.

DECLERCK, S.; STRULLU, D.G.; FORTIN, J.A. (Ed.). In vitro culture of mycorrhizas. Berlin: Springer-Verlag, 2005. v.4. p.73-91.
DOUDS JUNIOR, D.D.; NAGAHASHI, G.; PFEFFER, P.E.; REIDER, C.; KAYSER, W.M. On-farm production of AM fungus inoculum in mixtures of compost and vermiculite. Bioresource Technology, v.97, p.809-818, 2006.

FERREIRA, D.F. Sisvar: sistema de análise de variância. Lavras: Ufla, 2006.

FITTER, A.H. Darkness visible: reflections on underground ecology. Journal of Ecology, v.93, p.231-243, 2005.

GIANINAZZI, S.; VOSÁTKA, M. Inoculum of arbuscular mycorrhizal fungi for production systems: science meets business. Canadian Journal of Botany, v.82, p.1264-1271, 2004.

GIOVANNETTI, M.; MOSSE, B. An evaluation of techniques for measuring vesicular-arbuscular mycorrhizal infection on roots. New Phytologist, v.84, p.489-500, 1980.

MOHAMMAD, A.; KHAN, A.G.; KUEK, C. Improved aeroponic culture of inocula of arbuscular mycorrhizal fungi. Mycorrhiza, v.9, p.337-339, 2000.

MOREIRA, F.M. de S.; SIQUEIRA, J.O. Microbiologia e Bioquímica do Solo. 2.ed. atual. e ampl. Lavras: Ufla, 2006. $729 \mathrm{p}$.

NAIR, M.G.; SAFIR, G.R.; SIQUEIRA, J.O. Isolation and identification of vesicular-arbuscular mycorrhiza stimulatory compounds from clover (Trifolium repens) roots. Applied and Environmental Microbiology, v.57, p.434-439, 1991.

PARNISKE, M. Arbuscular mycorrhiza: the mother of plant root endosymbioses. Nature Reviews Microbiology, v.6, p.763-775, 2008.

PHILLIPS, J.M.; HAYMAN, D.S. Improved procedures for clearing roots and staining parasitic and vesicular-arbuscular mycorrhizal fungi for rapid assessment of infection. Transactions of the British Mycological Society, v.55, p.158-161, 1970.

POWELL, C.L.; BAGYARAJ, D.J. VA mycorrhiza. Boca Raton: CRC Press, 1984. 234p.

SCHUBERT, A.; LUBRACO, G. Mycorrhizal inoculation enhances growth and nutrient uptake of micropropagated apple rootstocks during weaning in commercial substrates of high nutrient availability. Applied Soil Ecology, v.15, p.113-118, 2000.

SILVA-JÚNIOR, J.P. da; SIQUEIRA, J.O. Aplicação de formononetina sintética ao solo como estimulante da formação de micorriza no milho e na soja. Revista Brasileira de Fisiologia Vegetal, v.9, p.33-39, 1997.

SILVA-JÚNIOR, J.P. da; SIQUEIRA, J.O. Colonização micorrízica e crescimento da soja com diferentes fungos e aplicação do isoflavonóide formononetina. Pesquisa Agropecuária Brasileira, v.33, p.953-959, 1998.

SIQUEIRA, J.O.; LAMBAIS, M.R.; STURMER, S.L. Fungos micorrízicos arbusculares: características, associação simbiótica e aplicação na agricultura. Biotecnologia Ciência e Desenvolvimento, v.25, p.12-21, 2002.

SIQUEIRA, J.O.; SAFIR, G.R.; NAIR, M.G. Stimulation of vesicular-arbuscular mycorrhiza formation and growth of white clover by flavonoid compounds. New Phytologist, v.118, p.87-93, 1991a.

Pesq. agropec. bras., Brasília, v.44, n.5, p.496-502, maio 2009 
SIQUEIRA, J.O.; SAFIR, G.R.; NAIR, M.G. VA-mycorrhizae stimulating and mycorrhiza isoflavonoid compounds reduce plant herbicide injury. Plant and Soil, v.134, p.233-242, 1991 b.

SOUZA, F.A. de; TRUFEM, S.F.B.; ALMEIDA, D.L. de; SILVA, E.M.R. da; GUERRA, J.G.M. Efeito de pré-cultivos sobre o potencial de inóculo de fungos micorrízicos arbusculares e produção da mandioca. Pesquisa Agropecuária Brasileira, v.34, p.1913-1923, 1999.

TSAI, S.M.; PHILLIPS, D.A. Flavonoids released naturally from alfalfa promote development of symbiotic Glomus spores in vitro. Applied and Environmental Microbiology, v.57, p.1485-1488, 1991.

Recebido em 11 de fevereiro de 2009 e aprovado em 30 de abril de 2009 